\title{
A high prevalence of glucokinase mutations in gestational diabetic subjects selected by clinical criteria
}

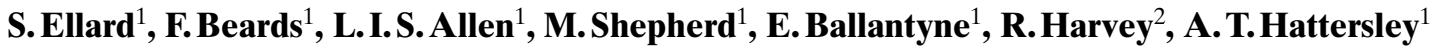 \\ ${ }^{1}$ Department of Vascular Medicine and Diabetes Research, School of Postgraduate Medicine and Health Sciences, \\ University of Exeter, Exeter, UK \\ ${ }^{2}$ Dr Gray's Hospital, Elgin, Morayshire, Scotland, UK
}

\begin{abstract}
Aims/hypothesis. Patients with glucokinase mutations are characterised by mild, persistent fasting hyperglycaemia, a small increment in glucose in response to an oral load and a dominant family history. These patients frequently present with gestational diabetes and often require insulin treatment during pregnancy. We assessed whether the selection of gestational diabetic subjects by clinical criteria would result in a high detection rate of glucokinase mutations. Methods. Caucasian gestational diabetic subjects from the United Kingdom who had fasting hyperglycaemia in pregnancy but did not meet the diagnostic criteria for maturity-onset diabetes of the young (MODY) were selected for direct sequencing of the glucokinase gene if they fulfilled the following four criteria; (1) persisting fasting hyperglycaemia outside pregnancy $(5.5-8 \mathrm{mmol} / \mathrm{l})$ (2) a small increment $(<4.6 \mathrm{mmol} / \mathrm{l})$ during a $2-\mathrm{h}$ oral glucose tolerance test (3) insulin treatment during at least one pregnan-
\end{abstract}

cy but subsequently controlled on diet and (4) a history of Type II (non-insulin-dependent) diabetes mellitus, gestational diabetes or fasting hyperglycaemia ( $>5.5 \mathrm{mmol} / \mathrm{l}$ ) in a first-degree relative.

Results. Of the 15 subjects $12(80 \%)$ with all these clinical criteria had glucokinase gene mutations. These included four previously unreported mutations $(\mathrm{N} 180 \mathrm{~K}, \mathrm{R} 191 \mathrm{~W}, \mathrm{Y} 215 \mathrm{X}$ and L288-1G $\rightarrow$ A).

Conclusion/interpretation. Phenotypic selection of subjects with gestational diabetes greatly increases the likelihood of detecting a mutation in the glucokinase gene as previous studies have suggested a prevalence of $2.5 \%$ (range $0-6 \%$ ). Our study in gestational diabetes to successfully used clinical criteria to assist in the definition of a genetic subgroup. [Diabetologia (2000) 43: 250-253]

Keywords Glucokinase, gestational diabetes, maturity-onset diabetes of the young, mutation analysis, phenotype selection.
Gestational diabetes mellitus (GDM) is a heterogeneous group of disorders in which diabetes or glucose intolerance is first diagnosed during pregnancy. Gestational diabetic patients include those with previously undiagnosed Type I (insulin-dependent) diabetes

Received: 5 August 1999 and in revised form: 1 October 1999

Corresponding author: Dr A.T.Hattersley, Department of Vascular Medicine and Diabetes Research, School of Postgraduate Medicine and Health Sciences, Barrack Rd, Exeter, EX2 5AX, UK

Abbreviations: GDM, Gestational diabetes mellitus; MODY, maturity-onset diabetes of the young. mellitus, Type II (non-insulin-dependent) diabetes mellitus, impaired glucose tolerance and specific defined monogenic subgroups in addition to pregnancy-induced glucose intolerance which reverses after delivery. The clinical course of patients with GDM varies considerably, both during and after pregnancy, reflecting the different causes.

Mutations in the glucokinase gene are a frequent cause of maturity-onset diabetes of the young (MODY) [1]. Despite the many different mutations described, the clinical phenotype is relatively constant. Patients with glucokinase mutations usually have mild $(5.5-8 \mathrm{mmol} / \mathrm{l})$ fasting hyperglycaemia throughout life $[1,2]$, with a small increment ob- 
served during an oral glucose tolerance test [3]. Accordingly, diabetic complications are rare and treatment with diet is usually adequate $[1,2,4]$. One exception is during pregnancy when insulin therapy is usually given [2].

Women with glucokinase mutations often present with gestational diabetes as their asymptomatic hyperglycaemia is detected by routine testing in pregnancy. Three series have suggested that the prevalence of glucokinase mutations in gestational diabetes is 5-6\% [5-7] but in other series mutations have not been detected [8-10]. It is important to identify these patients with glucokinase mutations because they have a predictable clinical course and the autosomal dominant inheritance means that each of their children has a $50 \%$ risk of being affected [4]. In contrast, the more common gestational diabetic subjects have normoglycaemic children, are themselves usually normoglycaemic immediately after pregnancy but at greatly increased risk of developing Type II diabetes in the subsequent 10-20 years.

Gestational diabetic subjects with glucokinase gene mutations often do not fulfil the diagnostic criteria for MODY. The mild hyperglycaemia associated with glucokinase mutations is dominantly inherited and occurs from birth but because mutation carriers are usually asymptomatic they might not be diagnosed before 25 years. In addition, even with OGTT testing more than $50 \%$ of mutation carriers do not reach glucose concentrations diagnostic of diabetes [1].

Routine mutation analysis of the glucokinase gene in all subjects with gestational diabetes is labour intensive because mutations are distributed throughout the coding region which consists of 12 exons. Selective genetic testing of patients with a high probability of having a glucokinase mutation on the basis of their clinical phenotype might be a more appropriate strategy. The aim of this study was to determine whether selecting gestational diabetic subjects (who do not meet the criteria for MODY) using criteria reported in the literature in subjects with glucokinase mutations would result in a high detection rate of glucokinase mutations.

\section{Subjects and methods}

Subjects. The study was approved by the local ethics committee and conducted in accordance with the Declaration of Helsinki principles.

Caucasian gestational diabetic subjects from the United Kingdom who had fasting hyperglycaemia $(5.5-8 \mathrm{mmol} / \mathrm{l})$ in pregnancy were selected for analysis of the glucokinase gene if they fulfilled the following four additional criteria: (1) persisting fasting hyperglycaemia outside pregnancy $(5.5-8 \mathrm{mmol} / \mathrm{l})[1,2,4](2)$ an increment between the fasting and 2-h plasma glucose concentrations of less than $4.6 \mathrm{mmol} / \mathrm{l}$ in at least one $75-\mathrm{g}$ oral glucose tolerance test (during or post pregnancy) [3] (3) insulin treatment during at least one pregnancy but subsequently controlled on diet [2, 4] (4) a history of Type II diabetes, gestational diabetes or fasting hyperglycaemia $(>5.5 \mathrm{mmol} / \mathrm{l})$ in a first-degree relative $[1,2,4]$. None of the subjects met the diagnostic criteria for MODY.

All subjects referred to our research unit by their physicians between 1995 and 1999 that met these criteria were included in this study. The majority of these patients came from diabetic pregnancy clinics in the South West of England and were referred as possibly having glucokinase mutations on the basis of their phenotype.

Genetic analysis. Genomic DNA was extracted from peripheral lymphocytes using a Nucleon DNA extraction kit (Scotlab, Coatbridge, UK). The coding regions of the 12 exons and the intron-exon boundaries of the glucokinase gene were amplified by PCR using published primer sequences. Polymerase chain reaction products were purified using QIAquick PCR purification columns (Qiagen, Crawley, UK) and both strands sequenced using a BigDye Terminator Cycle Sequencing kit (PE Biosystems, Warrington, UK) according to the manufacturer's recommendations. Reactions were analysed on an ABI Prism 377 DNA Sequencer (PE Biosystems).

\section{Results}

We recruited 15 subjects with gestational diabetes who satisfied all four criteria. Details of their clinical characteristics are shown in Table 1. In all families when the diagnosis of gestational diabetes was made in the proband no other family members had been diagnosed as having non-insulin dependent diabetes before the age of 25 years. This meant the families could not be diagnosed as having MODY. In one case (BDA31) the family was referred to us only when a child was found to have hyperglycaemia, but not diabetes, at the age of 4 years.

Direct sequencing of the glucokinase gene identified mutations in 12 of the $15(80 \%)$ gestational diabetic subjects. The K161+2del15 mutation was found in two subjects not known to be related. Four of these mutations have not been reported; N180K, $\mathrm{R} 191 \mathrm{~W}, \mathrm{Y} 215 \mathrm{X}$ and L288-1G $\rightarrow$ A. The L288-1G $\rightarrow$ A mutation alters the splice acceptor site of intron 7 and is predicted to result in a mRNA transcript lacking exon 8 or a frameshift mutation due to activation of a cryptic splice site. Glucokinase activity is likely to be severely reduced by both the L288-1G $\rightarrow$ A splice site and Y215X nonsense mutations. The novel missense mutations detected are likely to be mutations rather than polymorphisms because they altered the coding of an amino acid at sites conserved across species, cosegregated with a raised fasting blood glucose concentration in the family and were not present in at least 50 normal chromosomes. 
Table 1. Selection criteria and mutations detected in the glucokinase gene in subjects with gestational diabetes

\begin{tabular}{|c|c|c|c|c|}
\hline Patient reference & $\begin{array}{l}\text { Fasting glucose } \\
\text { after pregnancy mmol/l }\end{array}$ & $\begin{array}{l}\text { Smallest increment } \\
\text { on OGTT mmol/l }\end{array}$ & $\begin{array}{l}n \text { relatives fasting plasma } \\
\text { glucose }>5.5 \mathrm{mmol} / \mathrm{l}\end{array}$ & Mutation found \\
\hline BDA 07 & 6.7 & 2.8 & 1 & F150S (exon 4) \\
\hline BDA 31 & 6.5 & 1.0 & 2 & A259T (exon 7) \\
\hline BDA 50 & 7.0 & 2.6 & 1 & R403fsdelC (exon 9) \\
\hline BDA 57 & 6.0 & 2.5 & 1 & $\mathrm{~K} 161+2 \operatorname{del} 15$ (intron 4$)$ \\
\hline BDA 58 & 6.7 & 2.8 & 2 & N180K (exon 5) \\
\hline BDA 189 & 6.5 & 2.4 & 1 & $\mathrm{~K} 161+2 \operatorname{del} 15$ (intron 4) \\
\hline BDA 199 & 5.9 & 1.3 & 1 & E40ins21 (exon 2) \\
\hline BDA 224 & 6.4 & 1.6 & 4 & R191W (exon 5) \\
\hline BDA 286 & 5.9 & 2.8 & 2 & L288-1G > A (intron 7) \\
\hline BDA 67 & 6.1 & 1.1 & 2 & none \\
\hline BDA 76 & 6.3 & 2.6 & 5 & none \\
\hline
\end{tabular}

\section{Discussion}

Glucokinase gene mutations were detected in $80 \%$ of gestational diabetic patients selected on the basis of clinical phenotype. This is the highest reported frequency of glucokinase mutations in any series and is considerably higher than previously reported series of mutation screening in GDM subjects which have shown an overall frequency of $2.5 \%$ with mutations detected in only three [5-7] of the six studies [5-10]. The previously highest reported studies were $6 \%$ in British Caucasians [7], 5.9\% in French Caucasians [6], and 5\% in Americans [5]. These patients were, however, not completely non-selected as the British subjects were chosen for having persistent fasting hyperglycaemia on follow-up (>5.5 $\mathrm{mmol} / \mathrm{l})[7]$ and the American subjects had a first-degree relative with diabetes [5]. Our result suggests that careful selection of subjects on the basis of their clinical phenotype greatly increases the likelihood of detecting a mutation in the glucokinase gene. Conversely, there were patients with this strictly defined phenotype in whom mutations in the glucokinase gene were not found, hence genetic testing is still required for a definitive diagnosis.

This study uses a physician referred cohort so cannot be used to assess the likely prevalence of glucokinase mutations in GDM. In addition as we did not analyse a large number of subjects with varying numbers of the four diagnostic criteria we cannot assess their sensitivity and specificity. It is likely that some of these criteria are less discriminatory than others; the choice of treatment is physician determined as well as being a characteristic of the hyperglycaemia seen in the patient and there is considerable variation in the 2-h value of an OGTT. It is noticeable that the 2 -h increment measured in an oral glucose tolerance test was less than $3 \mathrm{mmol} / \mathrm{l}$ for all of our patients, suggesting that our standard of less than $4.6 \mathrm{mmol} / \mathrm{l} \mathrm{de}-$ fined by the previously reported 75 th centile [3] could be too high.
Phenotypic selection is frequently used in common cancers to limit genetic testing to those likely to belong to a monogenic subgroup. For example, mutation analysis of the $B R C A 1$ and $B R C A 2$ genes is usually offered only to families with multiple cases of early-onset breast or ovarian cancer or both, particularly when the breast cancer is bilateral. Another example of phenotype selection is that patients who fit criteria for MODY with a diagnosis before 25 years and an autosomal dominant inheritance are much more likely than patients with Type II diabetes to have mutations in the glucokinase [6] or $H N F-1 \alpha$ genes.

The identification of a glucokinase mutation in a patient with gestational diabetes is important for both the mother and the baby. The child has a $50 \%$ chance of inheriting the mutation and identification of this mutation could avoid the anxiety associated with an incidental finding of hyperglycaemia during childhood. In the absence of this knowledge, young children with mild fasting hyperglycaemia could be thought to be in the early stages of the development of Type I diabetes. A definite molecular genetic diagnosis through identification of a glucokinase mutation provides reassurance about the probable mild clinical course with a low risk of complications for both the mother and child [2].

We conclude that patients with gestational diabetes who have a persistently raised fasting glucose but a small increment during an OGTT, require insulin in pregnancy and have a first-degree relative with mild hyperglycaemia should be considered for mutation analysis of the glucokinase gene. Appropriate phenotypic selection based on clinical criteria will greatly increase mutation detection rates in this monogenic subgroup of diabetes.

Acknowledgements. We gratefully acknowledge the help of the subjects, their families and their referring doctors; Dr D. Matthews, Dr A. Millward, Dr T. Harvey, Dr K. Matyka, Dr 
D. Dunger, Dr A. Archer, Dr N. Lewis-Barned, Dr Mansell, Dr C. Close, Dr K. Macleod and Miss R. Sturley.

This work was funded by the Northcott Devon Medical Foundation, the British Diabetic Association, the University of Exeter and the Royal Devon and Exeter National Health Service Healthcare Trust.

\section{References}

1. Froguel P, Zouali H, Vionnet N et al. (1993) Familial hyperglycemia due to mutations in glucokinase. Definition of a subtype of diabetes mellitus. N Engl J Med 328: 697-702

2. Page RC, Hattersley AT, Levy JC et al. (1995) Clinical characteristics of subjects with a missense mutation in glucokinase. Diabet Med 12: 209-217

3. O'Rahilly S, Hattersley A, Vaag A, Gray H (1994) Insulin resistance as the major cause of impaired glucose tolerance: a self-fulfilling prophesy? Lancet 344: 585-589

4. Hattersley AT (1996) Glucokinase mutations and Type 2 diabetes. In: Lightman S (ed) Horizons in medicine. Blackwell Science, Bristol, pp 440-449
5. Stoffel M, Bell KL, Blackburn CL et al. (1993) Identification of glucokinase mutations in subjects with gestational diabetes mellitus. Diabetes 42: 937-940

6. Zouali H, Vaxillaire M, Lesage S et al. (1993) Linkage analysis and molecular scanning of glucokinase gene in NIDDM families. Diabetes 42: 1238-1245

7. Saker PJ, Hattersley AT, Barrow B et al. (1996) High prevalence of a missense mutation of the glucokinase gene in gestational diabetic patients due to a founder-effect in a local population. Diabetologia 39: 1325-1328

8. Chiu KC, Go RC, Aoki M et al. (1994) Glucokinse gene in gestational diabetes mellitus: population association study and molecular scanning. Diabetologia 37: 104-110

9. Allen CJ, Argyropoulos G, Bowker M et al. (1997) Gestational diabetes mellitus and gene mutations which affect insulin secretion. Diabetes Res Clin Pract 36: 135-141

10. Zaidi FK, Wareham NJ, McCarthy MI et al. (1997) Homozygosity for a common polymorphism in the islet-specific promoter of the glucokinase gene is associated with a reduced early insulin response to oral glucose in pregnant women. Diabet Med 14: 228-234 